\title{
Does informal online media provide supportive or unreliable information about pregnancy and birth during the COVID-19 pandemic?
}

\author{
Authors: Alexandra Cardoso Pinto, ${ }^{\mathrm{A}}$ Emily Southall, ${ }^{\mathrm{A}}$ Justyna Gromala ${ }^{\mathrm{A}}$ and Vivian Graf ${ }^{\mathrm{A}}$
}

Table 1. Accuracy and sentiment analyses as percentage of total within platform

\begin{tabular}{|c|c|c|c|c|c|}
\hline \multirow{2}{*}{\multicolumn{2}{|c|}{ Platform }} & \multirow{2}{*}{$\begin{array}{c}\text { Accuracy analysis } \\
\text { Percentage } \\
\text { inaccurate }\end{array}$} & \multicolumn{3}{|c|}{ Sentiment analysis } \\
\hline & & & $\begin{array}{c}\text { Percentage } \\
\text { negative }\end{array}$ & $\begin{array}{c}\text { Percentage } \\
\text { positive }\end{array}$ & $\begin{array}{c}\text { Percentage neutral } \\
\text { or both }\end{array}$ \\
\hline \multicolumn{2}{|c|}{ Twitter $(n=148)$} & 1 & 45 & 16 & 40 \\
\hline \multicolumn{2}{|c|}{ Facebook $(n=50)$} & 0 & 34 & 24 & 42 \\
\hline \multirow[t]{2}{*}{ Mumsnet } & Posts $(n=75)$ & 9 & 91 & 1 & 8 \\
\hline & Comments $(n=478)$ & 3 & 31 & 25 & 44 \\
\hline \multirow[t]{2}{*}{ News } & Title $(n=83)$ & - & 61 & 8 & 31 \\
\hline & Article $(n=84)$ & 14 & 48 & 11 & 41 \\
\hline
\end{tabular}

\section{Introduction}

$89 \%$ of mothers use social media for pregnancy and parenthood advice. ${ }^{1}$ It is essential that clinicians understand the information that patients read, in order to comprehend patients' views and concerns, and advise on which sources may be supportive, and which are unreliable.

Previous studies have also highlighted an ongoing infodemic, which may intensify fear and increase risk of deteriorating mental health. ${ }^{2}$

\section{Materials and methods}

This study assessed and compared content on online media platforms for sentiment analysis and factual accuracy of pregnancy/birth-related content during the COVID-19 pandemic.

Twitter, Facebook, Mumsnet and news outlets (BBC, Daily Mail, Guardian, Independent, The Sun and The Times) were chosen for analysis based on their popularity. Two categories of search terms were generated, one COVID-19 and one pregnancy-related. Inclusion criteria stipulated that posts were written in English, in the public domain, published between 23 March and 30 April 2020, and contained at least one term from each search category. Relevant search results were limited to a maximum of 10 per combination of terms. Results were

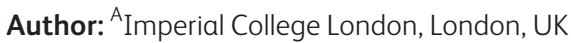

defined by category, source, sentiment and accuracy. Sentiment was determined as 'positive', 'negative', 'neutral' or 'both' by overarching emotion expressed, assessed independently by the authors, and an average was taken. Negative included fear, anger and disappointment, whereas positive included happiness, relief and gratitude. Accuracy was determined using the Royal College of Obstetricians and Gynaecologists' guidance issued in May, and was only measured for posts containing relevant information, which were a minority.

Significance of accuracy and sentiment comparisons were assessed using a Pearson's chi-squared test with Yates' continuity correction.

\section{Results and discussion}

Every platform had more negative than positive content. Mumsnet had a significantly higher percentage of negative posts than any other platform $(\mathrm{p}<2.20 \mathrm{E}-16)$; a majority of posts shared concerns and frustrations about experiences during COVID-19. There were fewer negative comments on Mumsnet than posts $(\mathrm{p}=2.20 \mathrm{E}-16)$; many provide neutral advice or positive support. In the news outlets, there were more negative titles than articles $(\mathrm{p}=0.10)$; the lack of significance indicates the consistency in sentiment. The proportion of neutral/both news articles was similar to that of posts on Twitter and Facebook $(p=0.10)$, perhaps because a large proportion of Twitter and Facebook posts were about, or included, news articles.

Percentage of overall inaccuracy was low. It was highest in news $(\mathrm{p}=1.36 \mathrm{E}-6)$, but all of these were outdated articles, accurate at the time of publication (Table 1). 


\section{Conclusion}

A high proportion of content analysed expressed negative sentiments, exposure to which may increase risk of depression and anxiety. ${ }^{2}$ Future studies should investigate how this can affect mothers' wellbeing and mental health.

This study found low levels of inaccuracy; while this may suggest these online media platforms are not unreliable sources of information about pregnancy/birth during COVID-19, this cannot be confirmed. Only a minority of posts contained guidance or information, suggesting online platforms are used more for sharing opinions or stories, of which accuracy was not determined. Therefore, increasing the presence of NHS-sourced information on online media could be beneficial, increasing access to reliable guidance.

\section{Conflicts of interest}

None declared.

\section{References}

1 Baker B, Yang I. Social media as social support in pregnancy and the postpartum. BMJ Sex Reprod Health 2018;17:31-4.

2 Chao M, Xue D, Liu T et al. Media use and acute psychological outcomes during COVID-19 outbreak in China. J Anxiety Disord 2020;74:102248. 